\title{
A cancer-specific transcriptional signature in human neoplasia
}

\author{
Francesco Nicassio, 1,2 Fabrizio Bianchi,,1,2 Maria Capra,, ${ }^{1,2}$ Manuela Vecchi, ${ }^{1,2}$ \\ Stefano Confalonieri, ${ }^{1,2}$ Marco Bianchi, ${ }^{1}$ Deborah Pajalunga, ${ }^{3}$ Marco Crescenzi, ${ }^{3}$ \\ Ian Marc Bonapace, 1,2,4 and Pier Paolo Di Fiore $1,2,5$
}

\begin{abstract}
${ }^{1}$ IFOM, Istituto Fondazione Italiana per la Ricerca sul Cancro di Oncologia Molecolare, Milan, Italy. ${ }^{2}$ Istituto Europeo di Oncologia, Milan, Italy. ${ }^{3}$ Department of Environment and Primary Prevention, Istituto Superiore di Sanità, Rome, Italy. ${ }^{4}$ Dipartimento di Biologia Strutturale e Funzionale, Universita' dell'Insubria, Varese, Italy. ${ }^{5}$ Dipartimento di Medicina, Chirurgia ed Odontoiatria, Universita' di Milano, Milan, Italy.
\end{abstract}

\begin{abstract}
The molecular anatomy of cancer cells is being explored through unbiased approaches aimed at the identification of cancer-specific transcriptional signatures. An alternative biased approach is exploitation of molecular tools capable of inducing cellular transformation. Transcriptional signatures thus identified can be readily validated in real cancers and more easily reverse-engineered into signaling pathways, given preexisting molecular knowledge. We exploited the ability of the adenovirus early region 1 A protein (E1A) oncogene to force the reentry into the cell cycle of terminally differentiated cells in order to identify and characterize genes whose expression is upregulated in this process. A subset of these genes was activated through a retinoblastoma protein/E2 viral promoter required factor-independent ( $\mathrm{pRb} / \mathrm{E} 2 \mathrm{~F}$-independent) mechanism and was overexpressed in a fraction of human cancers. Furthermore, this overexpression correlated with tumor progression in colon cancer, and 2 of these genes predicted unfavorable prognosis in breast cancer. A proof of principle biological validation was performed on one of the genes of the signature, skeletal muscle cell reentryinduced (SKIN) gene, a previously undescribed gene. SKIN was found overexpressed in some primary tumors and tumor cell lines and was amplified in a fraction of colon adenocarcinomas. Furthermore, knockdown of SKIN caused selective growth suppression in overexpressing tumor cell lines but not in tumor lines expressing physiological levels of the transcript. Thus, SKIN is a candidate oncogene in human cancer.
\end{abstract}

\section{Introduction}

Cancer-specific transcriptional signatures are being searched for identification of pathways subverted in neoplasia and to ameliorate patient management. These efforts are mainly concentrated on unbiased screening of cancer transcriptomes and have yielded, so far, important insights (see, e.g., refs. 1, 2). Yet one limitation of these approaches is the assumption of relative genetic homogeneity within the subpopulation of analyzed patients, an assumption that might not necessarily be true (see, e.g., refs. 3-5) Therefore, signatures identified through unbiased approaches might result in relatively "unstable" signatures, which in turn might decrease the reliability of the predictors once applied on a wider scale for diagnostic purposes. Furthermore, unbiased approaches are bound to identify the endpoints of complex cancer-causing alterations. The consequence of this is 2 -fold. On the one hand, the reverse engineering of the subverted molecular pathways might not immediately be obvious. On the other, because of the absence of temporal and mechanistic dimensions, end-point alterations might reflect

Nonstandard abbreviations used: $\mathrm{CBP}, \mathrm{CREB}-$ binding protein; CDK2, cyclindependent kinase 2; CtBP, C-terminal binding protein; CycA-E, cyclinA-E; E1A, early region $1 \mathrm{~A}$ protein; E2F, E2 viral promoter required factor; ER, estrogen receptor; FISH, fluorescence ISH; ISH, in situ hybridization; KD, knock down; Ki-67, antigen identified by monoclonal antibody Ki-67; MSC, muscle satellite cells; N0, lymph-node negative; pCAF, p300/CBP-associated factor-associated factor; $\mathrm{pRb}$, retinoblastoma protein; Q-RT-PCR, quantitative RT-PCR; siRNA, small interfering RNA; SKIN, skeletal muscle cell reentry induced; TD, terminally differentiated; TMA, tissue microarray; TRRAP, transformation/transcription domain-associated protein.

Conflict of interest: The authors have declared that no conflict of interest exists.

Citation for this article: J. Clin. Invest. 115:3015-3025 (2005).

doi:10.1172/JCI24862. equally well events that are pathogenetic in neoplasia or merely consequential to transformation.

These problems can be circumvented by exploiting biased approaches relying on molecular tools that cause transformation of cells in vitro. Based on the assumption that a limited number of altered signaling pathways lead to the malignant state, such devices can be employed as the starting point of screenings aimed at the identification of transformation signatures that can be subsequently validated against human cancers. The major advantage, in this case, is the availability of an in vitro model system that allows biochemical and genetic studies to reverse-engineer the pathways since both the starting and end-points are known.

To perform a biased approach to cancer transcriptomes, we utilized a model of terminally differentiated (TD) myotubes in vitro, induced to reenter the cell cycle by the early region $1 \mathrm{~A}$ protein (E1A) viral oncogene, an early gene product of tumorigenic adenovirus. Our choice was made for a number of concurring reasons.

First, TD myotubes constitute a close to physiological cellular model, and they are characterized by postmitotic cell cycle block and expression of differentiation markers (6). The withdrawal from the cell cycle is tightly regulated and insensitive to several well-characterized oncogenic stimuli. For instance, TD myotubes cannot be induced to DNA synthesis by overexpression of cell cycle positive regulators, such as $\mathrm{E} 2$ viral promoter required factor-family (E2F-family) transcriptional factors or active cyclinA-E/cyclindependent kinase 2 (CycA-E/CDK2) complexes $(7,8)$, or by knockout of cell cycle inhibitors, such as retinoblastoma protein $(\mathrm{pRb})$ or CDK-interaction protein 1, wild-type p53-activated fragment 1 (p21Cip/Waf) (9-11). Of note, the same stimuli are all capable of overcoming the growth arrest of primary fibroblasts $(11,12)$. 
A

\begin{tabular}{|r|c|c|c|c|c|}
\hline Class A & & $24 \mathrm{~h}$ & $24 \mathrm{~h}$ & & \\
\hline Acc. No. & Short name & YH47 & E2F & $\boldsymbol{R b}^{-}$ & Time \\
\hline NM_178683 & XTP1 & & $*$ & $*$ & EARLY \\
\hline NM_007633 & CCNE1 & & & & EARLY \\
\hline NM_009104 & RRM2 & & & & EARLY \\
\hline NM_009830 & CCNE2 & & & & EARLY \\
\hline NM_007891 & E2F1 & & N.A. & & EARLY \\
\hline
\end{tabular}

\begin{tabular}{|c|c|c|c|c|c|}
\hline Class B & & $24 \mathrm{~h}$ & $24 \mathrm{~h}$ & & \\
\hline Acc. No. & Short name & YH47 & E2F & $\mathbf{R b}^{-1}$ & Time \\
\hline NM_026115 & HAT1 & & & & EARLY \\
\hline NM_133815 & LBR & & $*$ & & EARLY \\
\hline NM_010931 & NP95 & & & & EARLY \\
\hline NM_177784 & MGC22679 & & & & EARLY \\
\hline NM_176972 & K1594 & & & & EARLY \\
\hline NM_016777 & NASPS & & & & EARLY \\
\hline NM_008568 & MCM7 & & & & EARLY \\
\hline NM_008565 & MCM4 & & & & EARLY \\
\hline NM_008567 & MCM6 & & & & EARLY \\
\hline
\end{tabular}

\begin{tabular}{|c|c|c|c|c|c|}
\hline Class C & & $36 \mathrm{~h}$ & $36 \mathrm{~h}$ & & \\
\hline Acc. No. & Short name & YH47 & E2F & $\mathbf{R b}^{-}$ & Time \\
\hline NM_171826 & C3orf4 & $*$ & & & LATE \\
\hline NM_011816 & G3BP2 & $*$ & & & LATE \\
\hline XM_129997 & TAF3 & $*$ & & & LATE \\
\hline NM_026149 & CML66 & & & & LATE \\
\hline XM_358357 & K0648 & & $*$ & & LATE \\
\hline NM_019666 & NSAP1 & & $*$ & & LATE \\
\hline NM_181278 & FLJ37562 & & & & LATE \\
\hline NM_019693 & BAT1 & & & & LATE \\
\hline NM_019550 & PTB2 & & & & LATE \\
\hline
\end{tabular}

\begin{tabular}{|c|c|c|c|c|c|}
\hline Class D & & $36 \mathrm{~h}$ & $36 \mathrm{~h}$ & & \\
\hline Acc. No. & Short name & YH47 & E2F & $\mathbf{R b}^{-1}$ & Time \\
\hline NM_021535 & SMU-1 & & & & LATE \\
\hline NM_031179 & SF3B1 & & & $*$ & LATE \\
\hline XM_130287 & Ch-TOG & & & & LATE \\
\hline NM_145959 & SKIN & & & & LATE \\
\hline NM_019828 & TRPC4AP & & & & LATE \\
\hline
\end{tabular}

B
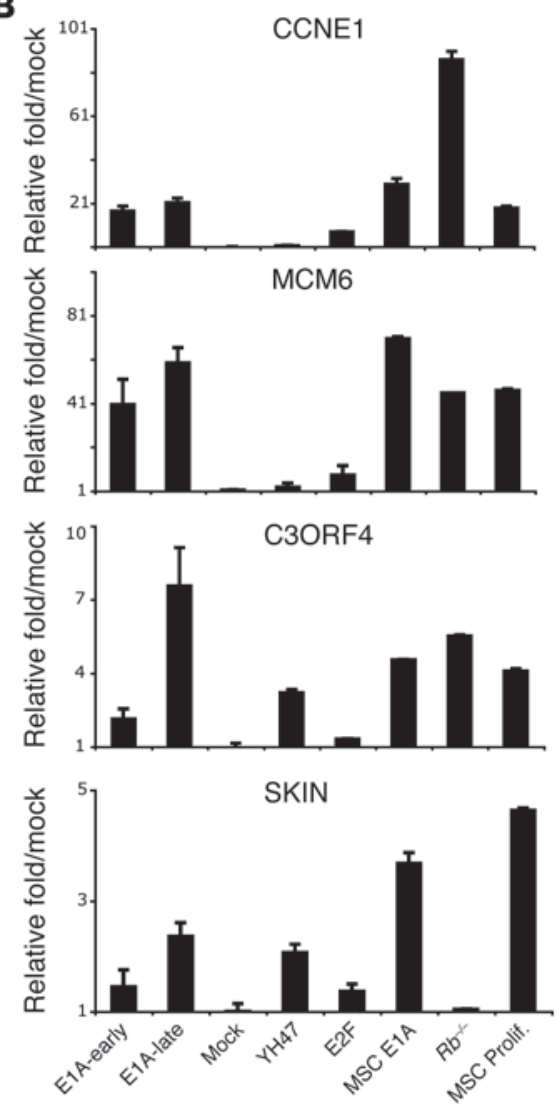

Figure 1

E1A-induced genes and their classification. (A) The E1A-induced genes are indicated by their accession numbers (acc. no.) and short names. Q-RT-PCR analysis was performed under the following conditions: (a) overexpression of E1A (not shown here; see Supplemental Table 2); (b) overexpression of the E1A mutant YH47/d1928 (YH47); (c) overexpression of E2F1 (E2F); and (d) removal of $R b\left(R b^{-/-}\right)$. Values were normalized to those obtained upon overexpression of E1A and are expressed with a color code. YH47 and E2F conditions, red/blue, $>$ or $<35 \%$ vs. the E1Ainduced condition, respectively; $R b^{-/-}$condition, red/blue, $>$or $<50 \%$ vs. the E1A-induced condition, respectively. Actual values are in Supplemental Table 4, and details on each experimental condition are in Supplemental Tables 5-7. Asterisks mark nonsignificant values $(P>0.05)$. Early and late refer to activation by E1A (actual values are in Supplemental Table 3). (B) Examples of data reported in A. The bar graphs show transcript levels (measured by Q-RT-PCR) of representative genes, as indicated. E1A-early and -late, overexpression of E1A at early and late time points; mock, dl312 adenovirus control; YH47, overexpression of the E1A mutant YH47/dl928; E2F, overexpression of E2F1; MSC-E1A, overexpression of $\mathrm{E} 1 \mathrm{~A}$ in MSCs; $R b^{-/-}$, removal of $R b$; MSC prolif., proliferating myoblasts. Values are normalized to mock-infected myotubes (assumed as 1.0). Results are from a single experiment; statistical analyses on repeated experiments are indicated in Supplemental Tables 2-7.

However, E1A can induce progression into S-phase and reversal of differentiation of TD myotubes $(7,13,14)$. Thus, we reasoned that the overcoming of such a stringent regulation might derive from E1A-induced mimicry of important proliferation pathways, which are by definition prime candidates for cancer pathways.

Second, while the action of E1A has been largely linked to its interference with the growth suppression function of pRb-family proteins (15-18), this event in itself is not capable of causing reentry into the cell cycle of TD myotubes $(9,10)$. Thus, additional E1A-induced pathways must be at work. Candidates for this function are E1A-regulated pocket protein/pRb-independent pathways, including those relying on CycA-E/CDK2, C-terminal binding protein (CtBP), transformation/transcription domainassociated protein (TRRAP), p400, PCAF-associated factor (pCAF) (PCAF, p300/CBP-associated factor; CBP, CREB-binding protein), and other chromatin remodeling activities (19-25). Even in more "canonical" in vitro systems of transformation, such as rodent embryo fibroblasts, pathways other than the $\mathrm{pRb}$ pathway have been linked to E1A oncogenic potency (21, 22, 26-29).

In human cancers, alterations of the $\mathrm{pRb}$ pathway are a frequent occurrence. However, they are thought to be insufficient by themselves to cause neoplasia, as also shown by recent evidence in a mouse retinoblastoma model (30-32). Thus, E1A might provide a useful tool to uncover pathways, needed along with the inactivation of the $\mathrm{pRb}$ pathway, which might be directly relevant to human carcinogenesis. The present studies were undertaken to validate this hypothesis.

\section{Results}

E1A-induced genes in TD myotubes. By a cDNA subtraction, we cloned genes whose expression is induced by E1A in the process of inducing TD myotubes to reenter the cell cycle (see Supplemental Figure 1; supplemental material available online with this article; doi:10.1172/JCI24862DS1). Twenty-eight genes 
A

\begin{tabular}{|c|c|c|}
\hline 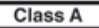 & Phase & Fold \\
\hline XTP1 & G2/M & \\
\hline CCNE1 & $\mathrm{s}$ & \\
\hline RRM2 & $\mathrm{s}$ & \\
\hline Class B & Phase & Fold \\
\hline NP95 & $\mathrm{s}$ & \\
\hline MG22679 & s & \\
\hline MCM4 & $\mathrm{s}$ & \\
\hline MCM6 & $\mathrm{s}$ & \\
\hline Class C & Phase & Fold \\
\hline C3orf4 & $=$ & \\
\hline CML66 & $=$ & \\
\hline K0648 & $=$ & \\
\hline FLJ37562 & $=$ & \\
\hline Class D & Phase & Fold \\
\hline SMU-1 & $=$ & \\
\hline SF3B1 & $=$ & \\
\hline Ch-TOG & G2/M & \\
\hline SKIN & $\equiv$ & \\
\hline TRPC4AP & $=$ & \\
\hline
\end{tabular}

B
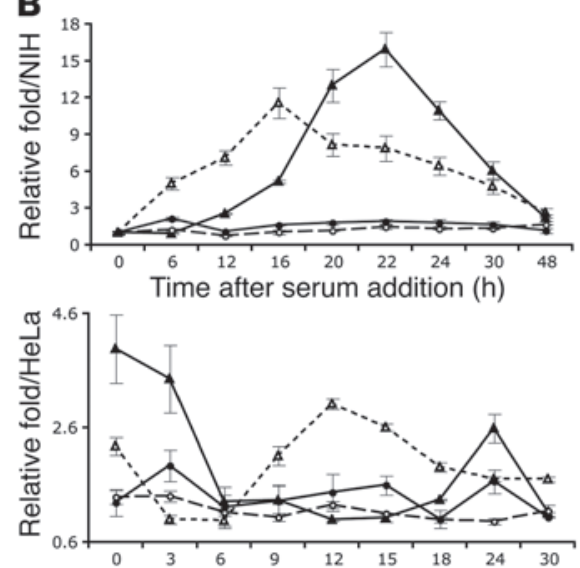

Time after nocodazole release (h)

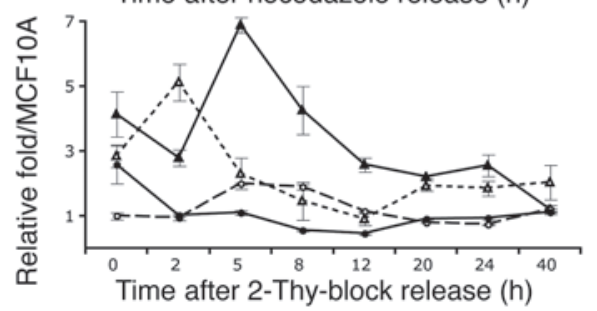

C

\begin{tabular}{|c|c|c|c|c|c|c|c|}
\hline Class A & \multicolumn{1}{|c|}{$\mathrm{t} 0$} & $\mathrm{t} 24$ & $\mathrm{t} 48$ & $\mathrm{t} 72$ & $\mathrm{t} 0 / \mathrm{t} 24$ & $\mathrm{t} 24 / \mathrm{t} 48$ & $\mathrm{t} 48 / \mathrm{t} 72$ \\
\hline XTP1 & 1.00 & 0.26 & 0.10 & 0.06 & & & \\
\hline CCNE1 & 1.00 & 0.30 & 0.13 & 0.12 & & & \\
\hline RRM2 & 1.00 & 0.73 & 0.65 & 0.48 & & & \\
\hline
\end{tabular}

\begin{tabular}{|c|c|c|c|c|c|c|c|}
\hline Class B & \multicolumn{1}{c|}{ t0 } & \multicolumn{1}{c|}{$\mathrm{t} 24$} & \multicolumn{1}{c|}{448} & \multicolumn{1}{c|}{$\mathrm{t72}$} & $\mathrm{t} 0 / \mathrm{t} 24$ & $\mathrm{t} 24 / \mathrm{t} 48$ & $\mathrm{t} 48 / \mathrm{t} 72$ \\
\hline NP95 & 1.00 & 0.20 & 0.07 & 0.02 & & & \\
\hline MCM4 & 1.00 & 0.21 & 0.06 & 0.02 & & & \\
\hline MCM6 & 1.00 & 0.36 & 0.09 & 0.05 & & & \\
\hline
\end{tabular}

\begin{tabular}{|c|c|c|c|c|c|c|c|}
\hline Class C & \multicolumn{1}{c|}{$\mathrm{t} 0$} & $\mathrm{t} 24$ & $\mathrm{t} 48$ & $\mathrm{t} 72$ & $\mathrm{t} 0 / \mathrm{t} 24$ & $\mathrm{t} 24 / \mathrm{t} 48$ & $\mathrm{t} 48 / \mathrm{t72}$ \\
\hline C3orf4 & 1.00 & 0.96 & 0.51 & 0.36 & & & \\
\hline CML66 & 1.00 & 1.18 & 0.87 & 0.35 & & & \\
\hline K0648 & 1.00 & 1.03 & 0.69 & 0.21 & & & \\
\hline FL37562 & 1.00 & 1.11 & 0.92 & 0.59 & & & \\
\hline
\end{tabular}

\begin{tabular}{|c|c|c|c|c|c|c|c|}
\hline Class D & t0 & t24 & \multicolumn{1}{|c|}{$\mathrm{t} 48$} & $\mathrm{t} 72$ & $\mathrm{t} 0 / \mathrm{t} 24$ & $\mathrm{t} 24 / \mathrm{t} 48$ & $\mathrm{t} 48 / \mathrm{t} 72$ \\
\hline SMU-1 & 1.00 & 1.33 & 0.94 & 0.65 & & & \\
\hline SF3B1 & 1.00 & 1.22 & 0.84 & 0.39 & & & \\
\hline Ch-TOG & 1.00 & 0.99 & 0.52 & 0.13 & & & \\
\hline SKIN & 1.00 & 0.87 & 0.40 & 0.16 & & & \\
\hline TRPC4AP & 1.00 & 1.05 & 1.15 & 0.97 & & & \\
\hline
\end{tabular}

Figure 2

E1A-induced genes in cell cycle and differentiation. E1A-induced genes were analyzed during cell cycle progression and MSC-differentiation. (A) Expression levels of representative genes, analyzed by Q-RT-PCR, during cell cycle progression of NIH-3T3. The lowest value in each kinetic was assumed as 1, and other values were normalized to it. Red, cell cycle regulated genes ( $>10$ fold); orange, poorly cell cycle regulated genes (4-10 fold); blue, not regulated, or marginally regulated, genes (marginally regulated, 2-4 fold: C3orf4, K0648, SF3B1; not regulated, <2 fold, CML66, FLJ37562, SMU-1, SKIN, and TRPC4AP). The cell cycle phase in which gene expression peaked is also indicated. (B) Examples of cell cycle regulation in NIH-3T3 (top), HeLa cells (middle), and MCF10A cells (bottom) of representative genes. TRPC4AP, open circles; SKIN, filled circles; XTP1, filled triangles; MGC22679, open triangles. (C) Representative genes were analyzed during differentiation of primary MSCs. MSCs were grown as proliferating myoblasts (t0) and then induced to differentiate. When committed to differentiation, myocytes undergo first an irreversible cell cycle arrest (characterized by block of DNA synthesis, p21 accumulation, and pRb hypophosphorylation) (t24), followed by phenotypic differentiation (t48) (evidenced by the accumulation of differentiation markers such as myosin heavy chain and muscle creatine kinase), and eventually by cell fusion (t72). Transcript levels were measured by Q-RT-PCR every 24 hours during differentiation and normalized to undifferentiated myoblasts (t0). Red indicates the time window in which the highest degree of regulation was detected.

(E1A-induced genes; Supplemental Table 1) showed reproducible and greater than 2-fold induction upon E1A expression in TD myotubes derived from C2C12 mouse myoblasts (Figure 1 and Supplemental Table 2). Importantly, comparable results were obtained when TD myotubes obtained from primary muscle satellite cells (MSCs) were used (Supplemental Table 2). Among the E1A-induced genes, 14 were early induced and 14 were late induced (Figure 1 and Supplemental Table 3). Almost all of the E1A-induced genes were actively transcribed in proliferating myoblasts but downregulated in myotubes, indicating that $\mathrm{E} 1 \mathrm{~A}$ action proceeds through the reactivation of programs switched off by terminal differentiation and withdrawal from the cell cycle (Supplemental Table 2).

Pathways leading to the activation of E1A-induced genes. E1A exerts pleiotropic effects on TD myotubes. It suppresses tissue-specific genes, through binding to the transcriptional coactivators p300/CBP, pCAF, and MyoD (33-36), and reactivates the cell cycle, through mechanisms involving binding to pocket proteins (mainly pRb and p130) and restoration of E2F activity $(15,35$, $37)$. However, the ectopic expression of E2Fs, or the targeted deletion of the $R b$ gene, in TD myotubes does not induce S-phase $(8-10)$, indicating that other E1A-activated pathways are also needed for this process (see Introduction). Thus, we tried to classify the E1A-induced genes according to their dependence on the pocket proteins/E2F pathway.

We employed 3 strategies in TD myotubes: (a) overexpression of E2F1, to identify genes whose induction is E2F1-dependent; (b) expression of an E1A mutant (YH47/d1928) that cannot bind to pocket proteins, to identify pocket protein-independent genes; and (c) removal of the $R b$ gene in TD myotubes derived from MSCs of $R b$-floxed mice (38). In the latter case, removal of $R b$ by Cre recombinase was obtained after the induction of terminal differentiation in an attempt to mimic those effects of E1A that are exclusively dependent on interference with $\mathrm{pRb}$. We analyzed the expression of the E1A-induced genes under these conditions in comparison with the levels obtained upon expression of E1A (Figure 1 and Supplemental Tables 4-7).

We identified 4 major classes of genes. Class A genes are pocket protein dependent (not induced or scarcely induced by YH47/ d1928; induced by $R b$ removal) and E2F1 dependent (induced by E2F1 overexpression). Class B genes are pocket protein dependent, E2F1-independent (or scarcely dependent). Interestingly, a subset of genes in this class (MCM 4, 6, and 7), which is known to be under the transcriptional control of E2F1 in nonpostmitotic cells 
A

\begin{tabular}{|l|c|c|c|c|c|c|c|c|c|c|}
\cline { 2 - 11 } \multicolumn{1}{c|}{} & Breast & Colon & Kidney & Larynx & Lung & Prostate & Stomach & Uterus & Melan. & Brain \\
\hline SF3B1 & & $*$ & & & $*$ & & & & & \\
\hline Ch-TOG & $*$ & $*$ & & $*$ & & & $*$ & & & \\
\hline SKIN & $*$ & $*$ & & $*$ & $*$ & & $*$ & & & \\
\hline TRCP4AP & & $*$ & & & $*$ & & $*$ & & & \\
\hline SMU-1 & & $*$ & & & & & & & & \\
\hline
\end{tabular}

B

SKIN: colon

SMU-1: breast
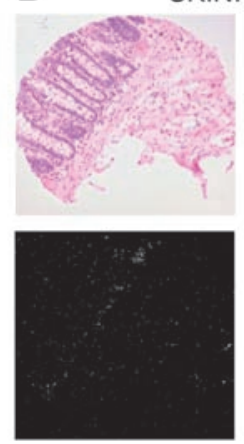

N
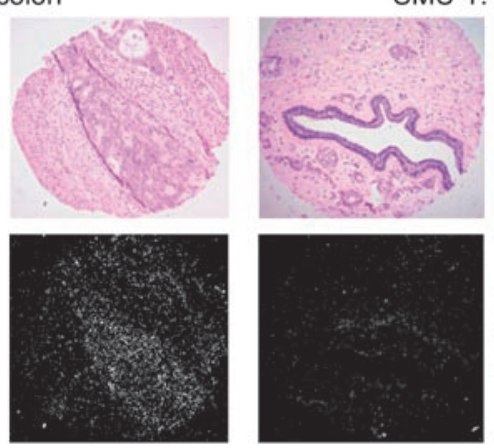

$\mathrm{T}$

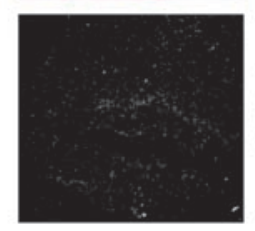

N

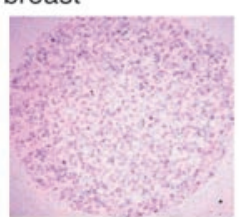

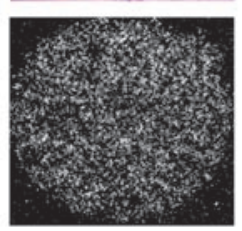

$\mathrm{T}$

TRCP4AP: lung
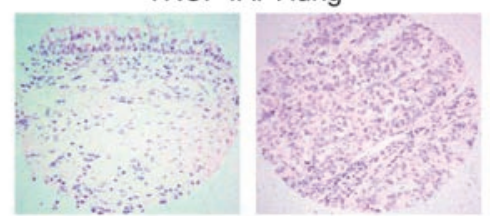

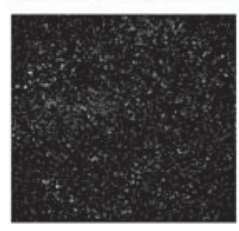

N

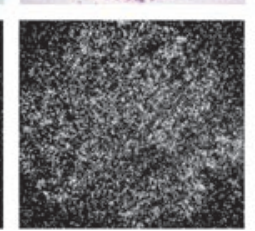

$\mathrm{T}$

C

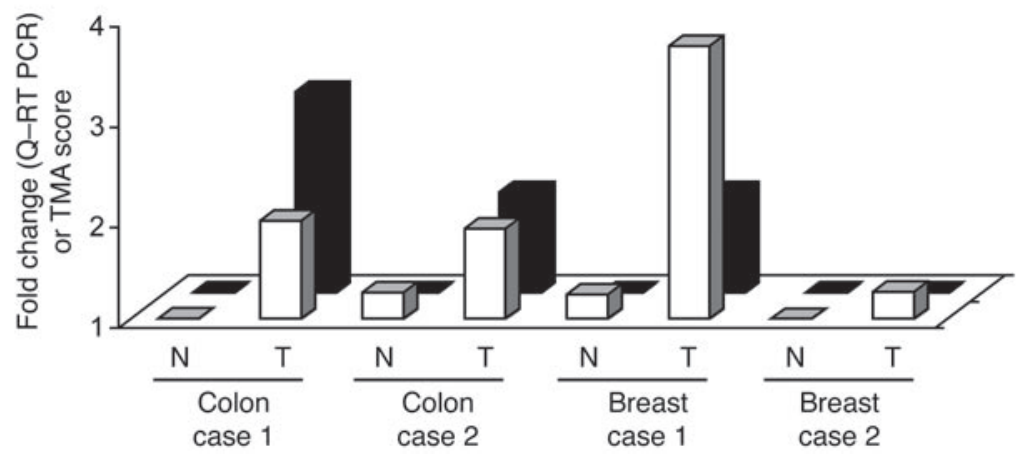

\section{Figure 3}

Class D genes in human tumors. (A) The overexpression of class D genes was tested by ISH on TMAs. The color code indicates the percentage of tumors in which overexpression was detected (actual numbers are reported in Supplemental Table 10). White, 0-20\%; blue, 21-40\%; green, 41-60\%; orange, 61-80\%; red, 81-100\%. In almost all cases (with the exception of TRPC4AP and SF3B1 in normal colon; see Figure 4), normal counterparts (not reported here) showed low or undetectable levels of expression ( $\leq 1$ on our scale; see Methods). Asterisks mark significant values $(P<0.05)$ of overexpression in tumors vs. normal counterparts. In some cases (uterus, melanoma [melan.], brain), statistical analysis could not be performed due to lack of normal tissues (see Supplemental Table 10 for further details). Eight other genes (from classes A-C, LBR, XTP1, MGC22679, K1594, C3orf4, CML66, K0648, and FLJ37562) showed no overexpression (not shown). Two additional class B genes (Np95 and Nasp) showed elevated mRNA levels in both tumors and proliferating cells of the normal counterparts, thus not fulfilling the criteria for overexpression (see text). (B) Examples of data reported in A. N, normal tissue; T, tumor. Top row, bright fields (histology); bottom row, dark fields (transcripts appear as bright dots). Original magnification, $\times 10$. (C) Tumor and normal samples from colon and breast carcinoma patients were analyzed for levels of expression of SKIN by ISH (black bars) and Q-RT-PCR (white bars), respectively. Q-RT-PCR data are normalized to colon case $1 \mathrm{~N}$ (assumed as 1.0). Data show good correlation between the 2 methods.

$(39,40)$, was not responsive to the overexpression of this protein in a TD environment, despite retaining pocket protein dependence. This suggests that there are differences in the transcriptional regulation of pocket protein/E2F-controlled genes in reversibly and irreversibly arrested cells, an issue that warrants further investigation. Class $\mathrm{C}$ genes are pocket protein indifferent (induced by YH47/d1928 but also activated by $R b$ removal). Almost all of these genes were E2F1 independent, with the exception of K0648. Class $\mathrm{D}$ genes are pocket protein independent or substantially independent. This group of 5 genes was activated by YH47/d1928 and scarcely by $R b$ removal. These genes were also E2F1 independent.

Cell cycle regulation of E1A-induced genes. To gain further insights into the cellular functions of the various classes of E1A-induced genes, we studied the regulation of 16 of them during the cell cycle. Progression into the cell cycle was analyzed in 3 different models: (a) serum-starved fibroblasts induced to reenter the cycle at the G0/G1 interface by serum addition; (b) nocodazole-synchronized HeLa cells induced to reenter the cycle from $M$ phase by nocodazole withdrawal; and (c) MCF10A, synchronized in S-phase by double thymidine block and released by addition of 2-deoxycytidine. In addition, we tested how these genes are regulated during the differentiation of $\mathrm{C} 2 \mathrm{C} 12$ myoblasts into myotubes.

Class A and B genes were all cell cycle-regulated (>10-fold induction) in fibroblasts, HeLa cells, and MCF10A cells (Figure 2, A and $B)$, with the majority of genes being induced at the G1/S transition (Figure 2, A and B). Similarly, they were immediately downregulated during differentiation concomitant to the withdrawal of cells from the cell cycle (Figure 2C). Together, these results suggest that class $\mathrm{A}$ and $\mathrm{B}$ genes are required for cell cycle progression, mainly for S-phase and DNA replication. 


\section{A}
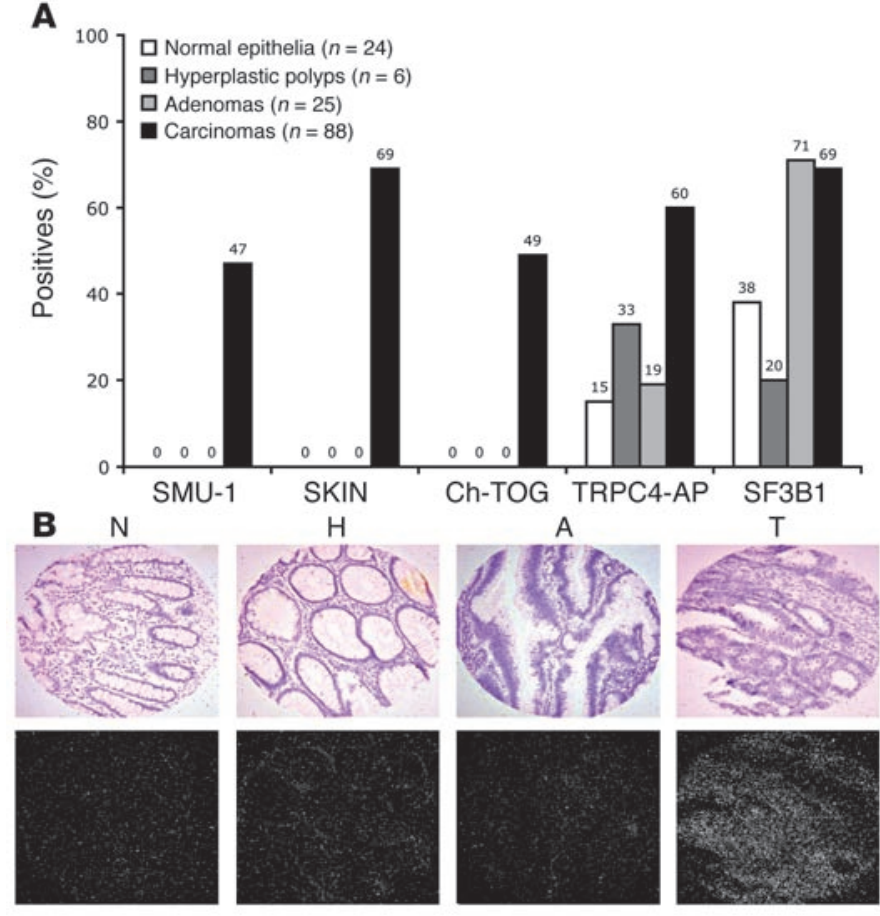

Ch-TOG TRF
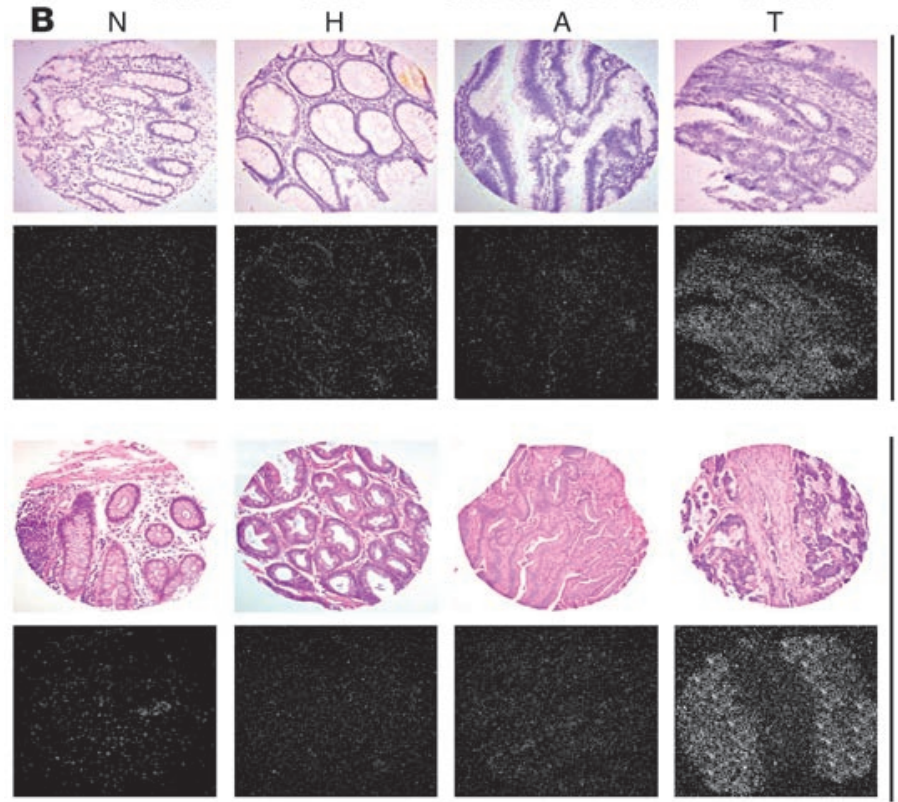

Figure 4

Class $D$ genes in colon cancer progression. (A) Expression of class $D$ genes was evaluated by ISH in the indicated samples on TMAs. Data are expressed as percentage of positive samples. The absolute number of positive samples is also indicated at the top of each column. (B) Selected examples of the data shown in A. N, normal epithelium; $\mathrm{H}$, hyperplastic polyp; A, adenoma; T, adenocarcinoma. Bright and dark fields are as in Figure 3. Original magnification, $\times 10$. See also Supplemental Table 12 for statistical analysis and correlations with clinical and biological parameters.

Class $\mathrm{C}$ and $\mathrm{D}$ genes, on the other hand, displayed either marginal ( $<4$-fold) or absent $(<2$-fold) regulation during the cell cycle in the 3 analyzed models (Figure 2, A and B). Moreover, during terminal differentiation, these genes were clearly downregulated at late stages of the process, well after the onset of the cell cycle block (Figure 2C). Thus, the E1A-induced regulation of these genes cannot be merely the consequence of the forced reentry of the TD myotubes in the cell cycle, but it is more likely part of the mechanism through which the postmitotic block is overridden by E1A-activated pathways.

Class D genes in human cancers. Our initial hypothesis was that a biased screening based on the ability of E1A to force reentry into the cell cycle of TD cells might lead to the identification and initial characterization of yet unknown cancer pathways. In keeping with this conjecture, the major goal of our quest was the identifi-
SMU-1

SKIN

cation of a set of genes induced by E1A through a pRb/ E2F-independent mechanism and whose induction by E1A could not be immediately interpreted as a "consequence" of the reentry of the myotubes in the cell cycle. Class D genes fulfill these requirements. Our hypothesis would further predict the involvement of the class D signature in human cancers.

We directly tested this possibility by in situ hybridization (ISH) on tissue microarrays (TMAs) (see Supplemental Table 8 for a validation of ISH-TMA as a reliable semiquantitative method). These TMAs contained hundreds of tumor samples derived from 10 different tumor types, frequently along with their matched normal counterparts (see Supplemental Table 9 for TMA composition and details of the analysis). Fourteen E1A-induced genes, from all classes, were tested. Genes from classes A, B, and C did not display overexpression in cancer samples. In particular, LBR, XTP1, MGC22679, K1594, C3Orf4, CML66, and FLJ37652 showed low or absent expression in both normal and tumor tissues (not shown). In 2 cases, Np95 and Nasp (both belonging to class B), the majority of tumor samples displayed high levels of expression (not shown). However, comparably high levels of expression were detected also in the proliferating cells of normal tissues (not shown). Thus Np95 and Nasp cannot be considered truly overexpressed, and their expression probably reflects simply the tumor hyperproliferative state (see Methods for the criteria used to define "overexpression").

Class D genes, conversely, were overexpressed in a fraction of cancers (Figure 3, A and B, and Supplemental Table 10). The pattern of expression of class $D$ genes displayed a clear tissue-specific dimension. As a group, class D genes were significantly overexpressed in colon cancer and to a lower extent in breast and lung tumors (Figure 3A and Supplemental Table 10). However, they were not overexpressed in kidney and prostate tumors (Figure 3A and Supplemental Table 10). Individual genes also displayed a tumor-specific pattern of overexpression; for instance, overexpression of skeletal muscle cell reentry-induced (SKIN) gene (a previously undescribed gene with the ability to encode an 838 amino acid-long protein; previously known as hypothetical protein FLJ23790; sequence submitted to GenBank, http://www.ncbi.nlm.nih.gov/entrez/query.fcgi?db=gene\&cmd) occurred at high frequency (> 70\% of the cases) in colon and larynx tumors and in melanomas, at intermediate/low frequency (20-30\%) in breast, lung, stomach, and uterus tumors, and at low or absent frequency $(0-10 \%)$ in kidney, prostate, and brain tumors. Oncomine (www.oncomine.org), a cancer microarray database, also revealed overexpression of some class D genes in certain tumors (Supplemental Table 11).

Finally, to confirm that results obtained by ISH-TMA allowed (semi)quantitative conclusions, we selected 2 matched pairs (tumor/normal) of breast and colon cancers (randomly from cases for which both frozen tissues and paraffin blocks were available) and compared the levels of SKIN by quantitative RT-PCR (Q-RT-PCR) and ISH-TMA. As shown in Figure 3C, both methods yielded comparable results. 
A

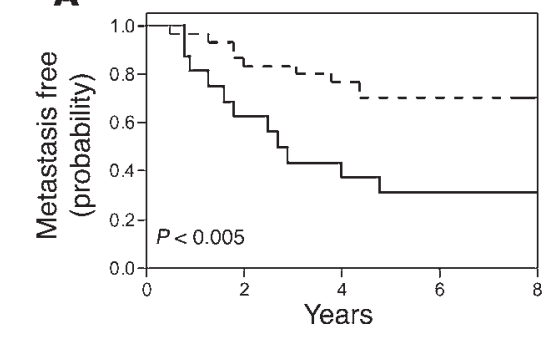

B

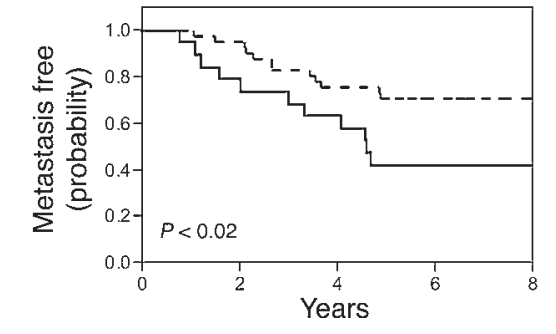

C

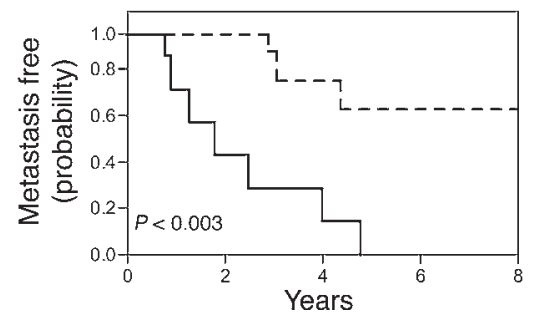

No. AT Risk

Years 0

Good 8

\section{Figure 5}

Selected class $D$ genes predict disease outcome in breast cancer. Two class D genes (SKIN and Ch-TOG) were used together as a predictor (see Supplemental Methods) of prognostic outcome on 2 independent data sets, one generated in-house (A), another from van't Veer (42) (B), and finally on 15 randomly selected breast tumor patients analyzed by Q-RT-PCR (C) (see Table 1 for details). Data are shown as the probability of remaining free of metastatic relapse, in a Kaplan-Meier plot, as a function of a favorable (dashed line) or unfavorable (continuous line) signature. See also Supplemental Table 13 for details. (C) Probability of remaining metastasis free is shown in a Kaplan-Meier plot, as a function of the presence of the favorable (dashed line) or unfavorable (continuous line) signature. In $\mathbf{A}-\mathbf{C}$, the $P$ values indicated were calculated with the log-rank test.

There is reason to think that the overexpression of class D genes is an event linked to the acquisition of the malignant phenotype rather than a consequence of transformation. First, class D genes were not generally expressed at high levels in normal proliferating epithelia. In the majority of cases, their expression was below detection in normal epithelial cells (see examples in Figure 3B) whereas in others (see below for TRPC4AP and SF3B1 in normal colonic mucosa), their level of expression was significantly lower than in the tumor tissue; in addition, they were homogeneously (> $80 \%$ of the cells) expressed in the tumor mass of the positive samples. Second, their pattern of overexpression was tumor specific (Figure 3A) and not a general property of all tested tumors. Third, there was no significant correlation between the expression of a tumor proliferation marker (Ki-67) and that of 4 of 5 class D genes (SKIN, SF3B1, SMU-1, and Ch-TOG), indicating that overexpression is not a consequence of the tumor hyperproliferative state (Supplemental Table 12). Only TRPC4AP correlated significantly with Ki-67 (Supplemental Table 12).

The initial analysis performed on TMAs indicated frequent alterations of class D genes in colon cancers. In these types of tumors, it is also possible to define progressive stages of cancer development. Thus, we analyzed a colon cancer progression TMA containing normal epithelia, hyperplastic polyps, adenomas, and adenocarcinomas. All 5 class D genes were overexpressed in $45-75 \%$ of adenocarcinomas. Three genes (SKIN, SMU-1, and ChTOG) were exclusively expressed in adenocarcinomas (Figure 4, A and B, and Supplemental Table 12) whereas TRPC4AP and SF3B1 were also expressed in normal epithelia and hyperplastic polyps, despite being detectable in tumors at higher frequency (Figure 4A and Supplemental Table 12) and with significantly higher levels of expression (not shown). Of note, SF3B1 was also overexpressed in adenomas (Figure 4A and Supplemental Table 12), consistent with the possibility that its overexpression represents a relatively early event in tumor progression.

Selected class $D$ genes predict disease outcome in breast cancers. Recent studies $(41,42)$ indicate that the acquisition of the metastatic potential is a rather early event in the natural history of tumors and that genetic signatures predictive of metastatic risk can be identified in primary tumors. Thus, we investigated a possible role of class D genes as predictors of metastatic risk in breast cancer. We extracted data regarding class $\mathrm{D}$ genes from microarray expression screenings performed on breast cancers. Two independent data sets were employed, 1 published by van't Veer and coworkers (42), and 1 generated by us, the complete results of which will be published elsewhere (see also Supplemental Table 13). In particular, we focused our attention on a subgroup of tumors with no lymph node involvement at surgery that either developed meta-

\section{Table 1}

Q-RT-PCR analysis of the 2 predictive class $D$ genes in breast cancer patients

\begin{tabular}{lcccc}
\hline Sample & ER & Group & SKIN & CH-TOG \\
1 & pos & NO & 1.00 & 1.00 \\
2 & pos & NO & 0.88 & 1.41 \\
3 & pos & NO & 1.05 & 1.15 \\
4 & pos & NO & 0.42 & 1.12 \\
5 & pos & NO & 0.91 & 0.86 \\
6 & pos & NO+ & 1.75 & 1.10 \\
7 & pos & NO+ & 3.05 & 1.63 \\
8 & pos & NO+ & 2.62 & 1.99 \\
9 & pos & NO+ & 1.87 & 2.85 \\
10 & pos & NO+ & 4.02 & 1.62 \\
11 & pos & NO+ & 0.96 & 1.18 \\
12 & pos & NO+ & 0.88 & 0.78 \\
13 & pos & NO+ & 2.56 & 2.93 \\
14 & pos & NO+ & 1.60 & 2.33 \\
15 & pos & NO+ & 1.22 & 1.51 \\
& $50 t h$ per. & & 1.05 & 1.41 \\
\hline
\end{tabular}

Q-RT-PCR analysis of the 2 predictive class $D$ genes was performed on 15 randomly selected breast tumor patients (all lymph node negative at diagnosis), which were all homogeneous for ER status (ER+). The relevant clinical data are summarized in the group column: N0, patients disease free for 5 years; $\mathrm{N} 0+$, patients relapsed with metastatic disease within 5 years. The values of Q-RT-PCR are shown normalized to patient 1 (assumed as 1.0). A 50th percentile value (50th per.) was then established for each gene (bottom row), and a matrix was built based on the 50th percentile value by assigning scores of 0 or 1 to values below or above the threshold, respectively. The sum of the 2 matrix scores was then used to assign favorable (score $0-1$ ) or unfavorable (score 2) labels. 
A

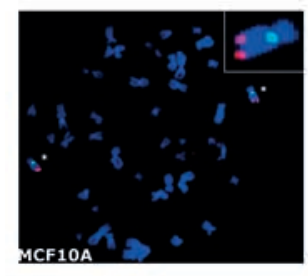

B Normal

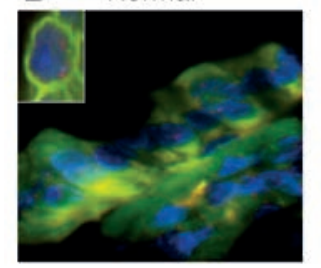

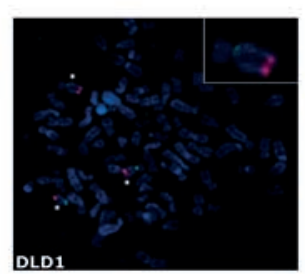

Tumor (not ampl.)

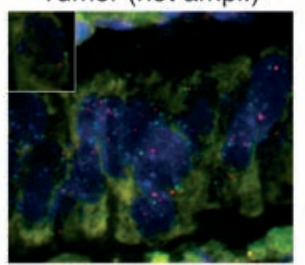

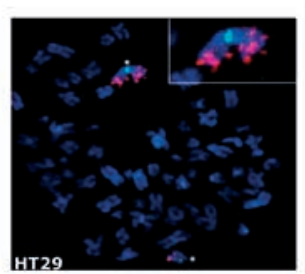

Tumor (ampl.)

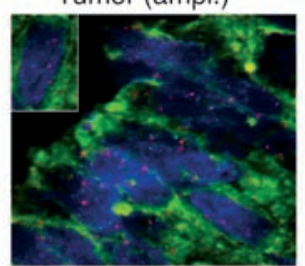

C

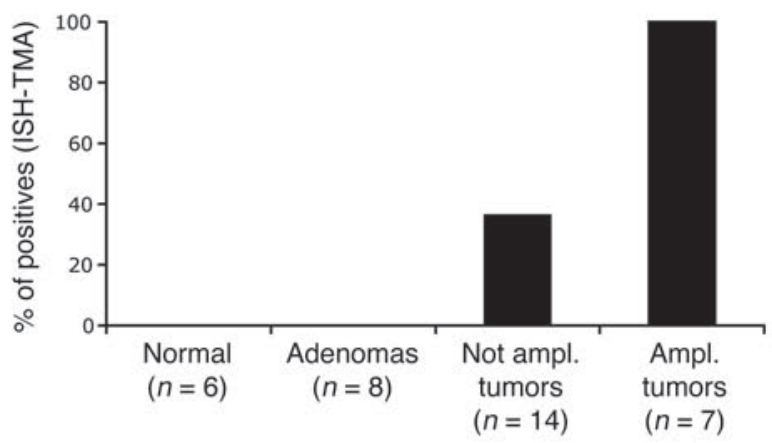

Figure 6

SKIN is amplified in colon cancers. (A) Left, FISH analysis on metaphase-blocked human cell lines (MCF10A, normal epithelial cells; DLD1 and HT29, colon carcinoma cells). Red, SKIN probe (RP11-1139F3); green, subcentromeric 8q probe (RP11-1031l1); blue, DAPI. Insets: Details of the corresponding images showing FISH on chromosome 8. Original magnification, $\times 100$. (B) FISH analysis in human colon cancer specimens of SKIN (red) and chromosome 8 (green). The average number of SKIN signals per cell was counted and normalized to the number of signals with chromosome 8 probe. Samples were considered amplified (amp.) if more than $50 \%$ of the epithelial cells presented exhibited more than 4 signals per cell. Examples are shown: normal, normal epithelium (copies/cell = 2); tumor, not amplified (copies/cell < 4); tumor, amplified (copies/cell > 4). Insets: Details of the corresponding images showing FISH on a single cell. Original magnification, $\times 60$. (C) The bar graph shows the percentage of SKIN-overexpressing samples (evaluated by ISH) in various colon specimens.

static disease or stayed disease free over a 5-year follow-up period. Two class D genes (SKIN and Ch-TOG) were able to predict the risk of disease relapse with approximately $70 \%$ accuracy (Figure 5, A and B; see also Supplemental Methods and Supplemental Figure 2). The predictive strength of the 2-gene model was further confirmed by Q-RT-PCR (Figure 5C and Table 1; see also Supplemental Methods and Supplemental Figure 2).

SKIN is amplified in colon cancer, and its functional ablation reduces proliferation of overexpressing cells. To further investigate the role of class D genes in human cancer, we focused, as a proof of principle, on SKIN, the class D gene that showed the most consistent and solid behavior in all the above-described characterizations. SKIN is a previously undescribed gene with the ability to encode an 838 amino acid-long protein (not shown), highly conserved in evolution (Supplemental Figure 3). One additional characteristic of SKIN is that the predicted protein is completely "anonymous" and does not display any known signature of functional and/or structural domains (not shown).

We searched for genetic alterations at the SKIN locus. Since overexpression is frequently due to increased gene dosage, we focused on SKIN gene amplification. Initially, we screened several cell lines by fluorescence ISH (FISH) on metaphase-blocked cells. Multiple SKIN-specific signals were detected in several tumor cell lines (Figure 6A and Table 2) independently of their state of aneuploidy. Moreover, SKIN amplification correlated well with its overexpression in the same cell lines, both at mRNA and protein levels (Figure 6A, Table 2, and Supplemental Figure 4). Next, we analyzed SKIN amplification directly on tumor tissues by in situ interphase FISH on colon specimens. In 6 samples of normal colonic mucosa and in 8 adenomas, no amplification (and no overexpression, as judged by ISH) of SKIN was detected (Figure 6B). In colon carcinoma, SKIN amplification (>4 signals/cell) was detected in 7 of 21 cases (33\%) (Figure 6B). Amplification was restricted to the epithelial components of the sample (Figure 6B). In all cases SKIN amplification was accompanied by overexpression judged by ISH (Figure 6B). Interestingly, in a sizable fraction of nonamplified cases $(6 / 14$, corresponding to $29 \%$ of all analyzed cases), overexpression of SKIN was detected by ISH (Figure 6B). Thus, SKIN overexpression can occur in the presence or absence of gene amplification, a situation reminiscent of that of other well-established oncogenes, such as ErbB-2 (43).

\section{Table 2}

Summary of SKIN amplification data on human cell lines

\begin{tabular}{lcccc} 
Cell line & RNA level & Copies & Chr. 8 & Ploidy \\
MCF10A & $1.04 \pm 0.2$ & 4 & 2 & 2 \\
WI38 & $1.22 \pm 0.0$ & 4 & 2 & 2 \\
HMEC & $2.03 \pm 0.2$ & 4 & 2 & 2 \\
SKMEL-28 & $1.56 \pm 0.1$ & $10-12$ & 5 & 2 \\
DLD-1 & $1.89 \pm 0.1$ & 6 & 3 & 2 \\
SW-480 & $2.47 \pm 0.1$ & 8 & 4 & 2 \\
TE671 & $2.91 \pm 0.2$ & $8-10^{\mathrm{A}}$ & 3 & $3-4$ \\
COL0 800 & $4.25 \pm 0.2$ & $6^{\mathrm{A}}$ & 2 & $>3$ \\
MDA-MB-361 & $4.31 \pm 0.2$ & $5^{\mathrm{A}}$ & 3 & $>3$ \\
MCF7 & $4.77 \pm 0.4$ & $>10^{\mathrm{A}, \mathrm{B}}$ & 4 & $>4$ \\
SKMEL-5 & $4.84 \pm 0.1$ & $12-14^{\mathrm{C}}$ & 6 & $>5$ \\
HT-29 & $9.26 \pm 0.9$ & $4^{\mathrm{C}}$ & 2 & $>6$ \\
SKBR3 & $9.64 \pm 0.6$ & $8^{\mathrm{A}, \mathrm{B}, \mathrm{C}}$ & 3 & $>6$ \\
\hline SUmmary & & & & \\
\hline
\end{tabular}

Summary of amplification data on cell lines. RNA level, SKIN transcripts measured by Q-RT-PCR and normalized to values in MCF10A cells (see Supplemental Figure 4 for details); copies, number of signals with the SKIN RP11-1139F3 probe; chr. 8, number of signals with the 8q RP11-1031l1 probe; ploidy, ratio between SKIN signals and chr. 8 signals. In the column indicating copies, additional features are marked as follows: Atandem repeats; Bextrachromosomal copies, Chsr (homogenously stained region). 

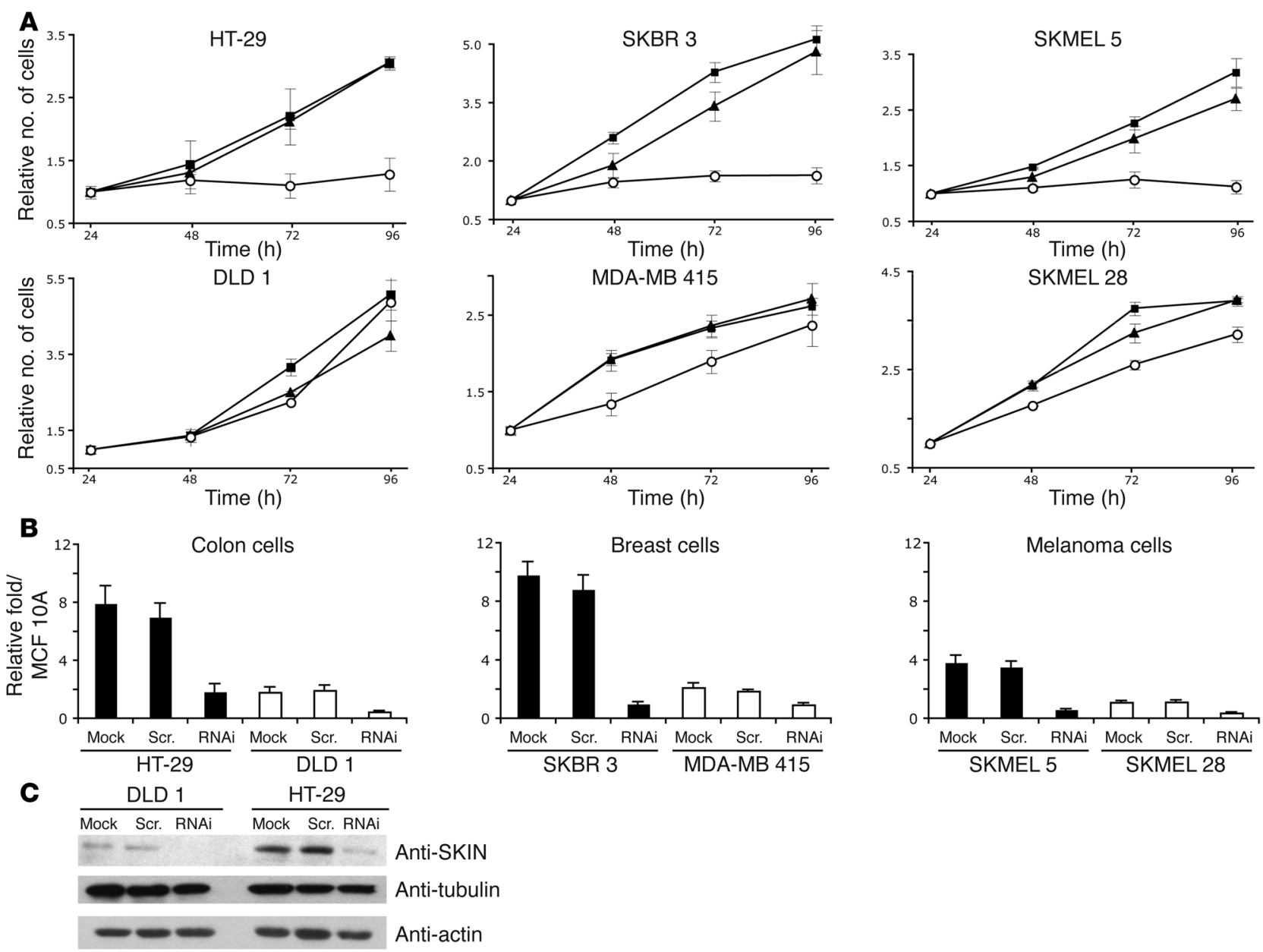

Figure 7

Functional ablation of SKIN in tumor cell lines. Six different tumor cell lines (as indicated) were treated with SKIN-specific siRNA (open circles in $\mathbf{A}$; RNAi in B and $\mathbf{C}$ ) or a control scrambled oligo (filled triangles in $\mathbf{A}$; scr. in $\mathbf{B}$ and $\mathbf{C}$ ) or were mock treated (filled squares in $\mathbf{A}$; mock in $\mathbf{B}$ and $\mathbf{C}$ ). Twenty-four hours after treatment, cells were replated to measure cell growth (A) or analyzed for SKIN transcript levels by Q-RT-PCR (B). (A) Cells, replated in standard growth medium, were counted at the indicated time points. Data are expressed relative to the number of cells present in the plate 24 hours after replating (assumed as 1). (B) Q-RT-PCR data are expressed relative to those detected in growing MCF10A cells, to allow for comparison among cell lines. The mRNA levels of 2 double-stranded RNA-activated protein kinase-induced (PKR-induced) genes (STAT1 and interferon-induced transmembrane protein-IFITM1) were also analyzed to exclude nonspecific effects driven by SKIN-siRNA procedures and are reported on in Supplemental Figure 6. (C) In the case of DLD1 and HT-29 cells, levels of SKIN were also measured by Western blot with an anti-SKIN antibody. Results in A, B, and C were also replicated with a second SKIN-specific siRNA (oligo 2; see Supplemental Methods) with comparable results (not shown).

The frequent genetic alterations at the SKIN locus predict a mechanistic involvement of this gene in malignant transformation. If so, SKIN overexpression should confer a proliferative advantage to the cell, and its functional ablation should revert this phenotype. In order to test this possibility, we selected, from a screening of several tumor cell lines (Figure 7A and Supplemental Figure 4), 6 cell lines to perform SKIN knockdown (KD) by small interfering RNA (siRNA). Three of the cell lines (HT-29, SKMEL5, and SKBR3) displayed SKIN overexpression (Figure 7, $B$ and C, and Supplemental Figure 4). Three other tumor cell lines (DLD1, SKMEL28, and MDA-MD415) showed normal levels of SKIN expression (Figure 7, B and C, and Supplemental Figure 4). Of note, tumor cell lines were selected to represent matched samples (overexpressing/not overexpressing) from the same types of tumors: colon carcinoma (HT29 and DLD1), mela- noma (SKMEL28 and SKMEL5), and breast carcinoma (SKBR3, and MDA-MB-415). As shown in Figure 7A, the KD of SKIN expression by siRNA dramatically reduced proliferation of all the overexpressing cell lines while a control scrambled oligo had little, if any, effect (additional controls are shown in Supplemental Figure 5). Importantly, SKIN KD did not inhibit proliferation of tumor lines displaying no overexpression (Figure 7A). Thus, SKIN is a candidate novel oncogene.

\section{Discussion}

Data presented in this study validate the hypothesis that a biased screening of transcriptomes in cancer-relevant in vitro models might lead to the identification of previously unknown putative cancer genes and pathways. By its ability to overcome the stringent regulation of withdrawal from the cell cycle in TD 
myotubes, E1A afforded a powerful tool to pursue these goals. In the set of genes induced by E1A, 2 genetic clusters could be readily identified. The first, comprising class A and B genes, is constituted by "typical" pocket-dependent E1A-responsive genes, whose transcription is induced either through E2F-dependent or E2F-independent mechanisms. A second genetic cluster (classes $\mathrm{C}$ and D) is made up of pocket-indifferent/independent genes. Within this cluster, class D genes fulfill the requirements of our quest, i.e., they constitute a signature, induced by a well-defined genetic alteration through a yet unknown mechanism. Indeed, class $\mathrm{D}$ genes could be implicated in a fraction of human cancers by a variety of approaches.

One relevant question is whether the signature identified by class D genes is causally involved in the determination of the malignant state or simply represents a read-out, or a consequence, of the process. The sum of our clinical/pathological and functional data argues in favor of the former possibility. First, the expression of class D genes was not (or was only marginally) cell cycle regulated in cell lines, supporting the notion that their overexpression in tumors cannot be the mere consequence of the tumor hyperproliferative state. This was further confirmed by the lack of correlation between the Ki-67 proliferative marker and the levels of class D gene expression (with the exception of TRPC4AP) in human tumors.

Second, the overexpression of class D genes correlated rather stringently with tumor progression in colon carcinoma, again suggesting a role in the determination of the malignant phenotype and in the progression of the natural history of the tumor.

Third, 2 selected class D genes were able to predict metastatic risk in breast carcinoma with approximately $70 \%$ accuracy in 2 independent case collections and datasets. The strength of this predictor is surprising because of the limited number of genes necessary to classify the patients compared with other published predictors (see, e.g., refs. 41, 42, 44). This is most likely due to the fact that our predictor was obtained through a biased cancer biology-driven approach, in which genetic noise and nonobligatory consequences of the transformation process were filtered out. Conversely, predictors obtained through unbiased approaches conceivably represent an average of the many steady states achievable in cancer cells.

Finally, direct evidence for genetic alterations affecting the SKIN locus, 1 of the class D genes, could be obtained in colon cancers. Furthermore, we point out the highly specific effects of the SKIN KD. SKIN expression could be efficiently knocked down in both overexpressing cells and cells expressing physiological levels of transcript. However, inhibition of proliferation could be detected only when SKIN was targeted in overexpressing cells. This demonstrates that overexpression of SKIN participates to the proliferative advantage of the overexpressing cell and argues for its causal involvement in cancer.

The involvement of class D genes in human cancers vis-à-vis their inducibility by E1A opens interesting scenarios at the genetic level. On the one hand, it is possible that in a fraction of human cancers a signaling pathway is constitutively activated, which leads to the transcription of class $\mathrm{D}$ genes. If so, the precise molecular knowledge of both the starting and the end points of the identified pathway (E1A and class D genes, respectively) should now allow the identification of the cancer-specific genetic alteration(s), which are predicted to lie in a pathway activated by E1A but independent of $\mathrm{pRb}$ and E2Fs. Obvious candidates for this role are genes encoding proteins such as CycA-E/CDK2, CtBP, pCAF, TRRAP, or p400 and their downstream signaling pathways, which are all activated by E1A independently of pRb/E2F (19-22, 24, 25). However, if the overexpression of class $\mathrm{D}$ genes is in itself sufficient to confer a proliferative advantage, as indicated at least in the case of SKIN by the KD experiments, then genetic cancer-determining alterations might affect directly class $\mathrm{D}$ gene loci. This second possibility, which does not preclude the first one, is again supported by our studies on SKIN, which was found amplified in a significant fraction of colon carcinomas.

Do class D genes represent separate end points of many signaling pathways concomitantly activated by E1A, or do they constitute elements of some molecular machinery with a defined cellular function? Class D genes encode rather heterogeneous proteins, including proteins involved in RNA splicing (SF3B1 and SMU-1; refs. 45-47), a microtubule-associated protein (Ch-TOG; refs. 48, 49), a component of the TNF-R1 pathway leading to activation of NF-KB (TRPC4AP; ref. 50), and a protein displaying no distinguishing features (SKIN). This heterogeneity cannot be immediately reconciled in a unifying scenario and apparently would favor the "many pathways" possibility. We note, however, that recent results unexpectedly involved ribonuclear complexes containing splicing factors and RNA-binding proteins in cytoskeletal regulation leading to cell adhesion (51). Thus, it is also possible that we might have identified a cluster of genes whose regulation is important in determining phenotypes frequently altered in cancer, such as cell adhesion to the substrate and motility: a possibility deserving additional experimental attention.

\section{Methods}

Cells, viruses, and cell biology procedures. C2C12 myoblasts, cultured in collagen-coated dishes in DMEM with $10 \%$ FBS, were induced to differentiate by serum deprivation for 72 hours in the presence of $50 \mathrm{mM}$ 1-D-arabinofuranosylcytosine, as described (35). Primary $\mathrm{Rb}^{\text {loxP/loxp }}$ MSCs (MSC-Rb $b^{\text {loxp } / \text { loxP }}$ ) were isolated from 2-week-old mice of the $\mathrm{Rb} \mathrm{b}^{\text {loxP/loxP }}$ genotype (a kind gift from A. Berns and M. Vooijs, The Netherlands Cancer Institute, Amsterdam, The Netherlands). Animal studies were approved by the Department of Veterinary Public Health, Italian Ministry of Health (Rome, Italy).

NIH-3T3 cells were synchronized by serum starvation for 48 hours followed by $10 \%$ FBS addition. HeLa cells were arrested in G2-M by nocodazole treatment $(50 \mathrm{ng} / \mathrm{ml})$ for 14 hours. MCF10A cells were synchronized at Sphase by double-thymidine $(2 \mathrm{mM})$ treatment for 16 hours followed by treatment with $10 \mu \mathrm{M} 2$-deoxycytidine. The cell cycle profile, at each time point, was monitored by FACS analysis.

Mutant or recombinant adenoviruses used for infections were as follows: d1520, expressing the $12 \mathrm{~S}$ form of E1A; YH47/d1928, expressing the $12 \mathrm{~S}$ form of a mutated E1A protein (Y47H, and C124G); and dl312, bearing a deletion of essentially the entire E1A gene (35). Recombinant Ad-E2F1 and Ad-CRE expressed their respective CDNAs under CMV promoter. A MOI of 300 was used in all cases except for Ad-CRE (MOI 1000).

SKIN reagents. Human and mouse SKIN complete cDNAs were reconstructed in silico based on the human (FLJ23790) and mouse (NM_ 145959) cDNA sequences and the Nucleotide-nucleotide BLAST est_ human and est_mouse databases (http://www.ncbi.nlm.nih.gov/blast/). We verified the $5^{\prime}$ sequence by performing $5^{\prime}$ rapid amplification of cDNA ends (RACE) on C2C12 myoblasts and WI38 RNAs with the RML-RACE kit according to the manufacturer's protocol (Ambion). The complete cDNA sequences of human and mouse SKIN are reported in Supplemental Methods. Mouse (1230) and Rabbit (si446) polyclonal antibodies against SKIN were raised using as immunogens the peptide 233-431 of mouse predicted protein and the peptide $406-838$ of human predicted 
protein, respectively, both produced as glutathione S-transferase (GST) fusion protein. The 2 sera recognized the same specific band, corresponding to an approximately $90-\mathrm{kDa}$ protein in human and mouse cell extracts. Western blot analysis of cells overexpressing a myc-tagged version of SKIN confirmed the antibody specificity.

FISH analysis. Cell lines were metaphase blocked with Colcemide $0.1 \mu \mathrm{g} / \mathrm{ml}$ (Invitrogen Corp.) for 2-6 hours. For FISH analysis, the following bacterial artificial chromosome clones were used: RP11-1139F3 and RP11-637L12 (SKIN, FLJ23790, 8q24.13); and RP11-1031I13 (chr. $8 \mathrm{q}$ sub-centromeric probe). Probes were labeled directly with CY3 and Fluor X (Amersham Biosciences) by nick translation. In situ FISH was performed with the Paraffin Pretreatment Kit II (Vysis) according to manufacturer's protocol. Gene copy number was evaluated by counting the average number of SKIN probe signals per cell, and normalized to the signal of the subcentromeric probe. At least 2 different fields were analyzed for each cell line or tissue sample. For the in situ FISH, samples were considered amplified only if more than $50 \%$ of the epithelial cells presented more than 4 signals per cell.

TMAs. Design and engineering of TMAs and ISH are described in Supplemental Methods and in Supplemental Table 9. Gene expression levels were evaluated by counting the average number of grains per cell and normalized to the signal of the housekeeping gene $\beta$-actin. Two pathologists counted independently all tumor cells in each TMA core using a darkfield condenser (Zeiss) for the silver grains. Signals were expressed in a semiquantitative scale: 0 , no staining; $1,1-25$ grains, weak staining; 2 , 26-50 grains, moderate staining; and 3 , more than 50 grains, strong staining. For a gene to be considered overexpressed the following criteria had to be satisfied: expression greater than or equal to 2 in more than $80 \%$ of the tumor cells and less than or equal to 1 in its normal counterpart. Also, if a gene was expressed at high levels in the proliferating cells of the normal tissue (as in the case of Np95 and Nasp), it was not considered overexpressed, even in the presence of intense staining of the tumor tissue.

Microarray experiments and data analysis. We screened $46 \mathrm{lymph}$ nodenegative (N0), estrogen receptor-positive (ER+), breast cancer patients with Affymetrix HG-U133 chip A+B (description of the methodology available in Supplemental Methods). From this screening, we extracted data regarding all E1A-induced genes (relevant clinical information and relevant microarray data are in Supplementary Table 13). We also extracted data regarding class $\mathrm{D}$ genes from the data set published by van't Veer and coworkers (42). In this case, data regarding all N0/ER+ patients of the study ( 60 patients; only ER+ patients were considered to have a data set directly comparable to ours, which only included ER+ patients) were extracted. The procedure of data analysis is described in detail in Supplemental Methods.

SKIN RNAi. The cell lines depicted in Figure 7 were transfected twice (24hour interval) with $100 \mathrm{nM}$ SKIN-specific siRNA or a scrambled control oligo (Dharmacon) using Oligofectamine (Invitrogen Corp.). Cells were then replated into 12-well plates at 15,000 cells/plate (SKMEL 5 or SKMEL 28), 20,000 cells/plate (SKBR 3, MDA-MB 415), or 45,000 cells/plate (HT-29 or DLD1) and counted in duplicate every 24 hours. Sequences of all oligos are in Supplemental Methods.

Other methods. Details as to Q-RT-PCR, primers, and oligonucleotides are in Supplemental Methods. The procedures used to identify the E1Ainduced genes are in the legend to Supplemental Figure 1.

Statistics. Statistical significance ( $P$ values) of the analyses reported in Figure 1A and Supplemental Tables 2-7 was evaluated with unpaired Student's parametric $t$ test. For the analysis of TMA data, reported in Figure 3A and in Supplemental Table 10, and for the correlation analysis, reported in Supplemental Table 12, contingency table analysis with likelihood-ratio $\chi^{2}$ $\left(\mathrm{G}^{2}\right)$ (JMP IN 5.1) was employed.

\section{Acknowledgments}

We are indebted to Giovanni d'Ario for invaluable help with statistical analyses. We thank A. Berns and M. Vooijs for the Rb-loxP mice; Roberto Papait and Eros Lazzerini Denchi for discussions and for providing reagents; and M. Quarto and O. Malazzi and P. Gasparini for FISH experiments. We also thank the Sequencing Service at IFOM. The authors' work is supported by grants from AIRC (Italian Association for Cancer Research) and the Italian Ministry of Health (to P. Paolo Di Fiore and M. Capra); the Monzino Foundation and the European Community (VI Framework) (to P. Paolo Di Fiore); and Telethon Italy (to M. Capra).

Received for publication February 25, 2005, and accepted in revised form July 26, 2005.

Address correspondence to: Pier Paolo Di Fiore, IFOM, Istituto FIRC di Oncologia Molecolare, Via Adamello 1620139 Milan, Italy. Phone: 39-02-574303247; Fax: 39-02-574303231; E-mail: pierpaolo.difiore@ifom-ieo-campus.it. Or to: Ian Marc Bonapace, Dip. di Biologia Strutturale e Funzionale,Università dell'Insubria, Via A. da Giussano 12, 21052 - Busto Arsizio, Varese, Italy. Phone: 39-331-339452-05; Fax: 39-331-339459; E-mail: ian.bonapace@uninsubria.it.
1. Ramaswamy, S., et al. 2001. Multiclass cancer diagnosis using tumor gene expression signatures. Proc Natl. Acad. Sci. U. S. A. 98:15149-15154.

2. Ramaswamy, S., and Golub, T.R. 2002. DNA microarrays in clinical oncology. J. Clin. Oncol. 20:1932-1941.

3. Iafrate, A.J., et al. 2004. Detection of large-scale variation in the human genome. Nat. Genet 36:949-951

4. Jones, C., et al. 2004. Molecular cytogenetic identification of subgroups of grade III invasive ductal breast carcinomas with different clinical outcomes. Clin. Cancer Res. 10:5988-5997.

5. Pollack, J.R., et al. 2002. Microarray analysis reveals a major direct role of DNA copy number alteration in the transcriptional program of human breast tumors. Proc. Natl. Acad. Sci. U. S. A. 99:12963-12968.

6. Andres, V., and Walsh, K. 1996. Myogenin expression, cell cycle withdrawal, and phenotypic differentiation are temporally separable events that precede cell fusion upon myogenesis. J. Cell Biol.
132:657-666

7. Tiainen, M., et al. 1996. Terminally differentiated skeletal myotubes are not confined to G0 but can enter G1 upon growth factor stimulation. Cell Growth Differ. 7:1039-1050.

8. Pajalunga, D., et al. 1999. E2F activates late-G1 events but cannot replace $\mathrm{E} 1 \mathrm{~A}$ in inducing $\mathrm{S}$ phase in terminally differentiated skeletal muscle cells. Oncogene. 18:5054-5062.

9. Huh, M.S., Parker, M.H., Scime, A., Parks, R., and Rudnicki, M.A. 2004. Rb is required for progression through myogenic differentiation but not maintenance of terminal differentiation. J. Cell Biol. 166:865-876

10. Camarda, G., et al. 2004. A pRb-independent mechanism preserves the postmitotic state in terminally differentiated skeletal muscle cells. J. Cell Biol. 167:417-423.

11. Deng, C., Zhang, P., Harper, J.W., Elledge, S.J., and Leder, P. 1995. Mice lacking p21CIP1/WAF1 undergo normal development, but are defective in G1 checkpoint control. Cell. 82:675-684.
12. Sage, J., Miller, A.L., Perez-Mancera, P.A., Wysocki, J.M., and Jacks, T. 2003. Acute mutation of retinoblastoma gene function is sufficient for cell cycle re-entry. Nature. 424:223-228.

13. Kirshenbaum, L.A., and Schneider, M.D. 1995. Adenovirus E1A represses cardiac gene transcription and reactivates DNA synthesis in ventricular myocytes, via alternative pocket protein- and p300binding domains. J. Biol. Chem. 270:7791-7794.

14. Crescenzi, M., Soddu, S., and Tato, F. 1995. Mitotic cycle reactivation in terminally differentiated cells by adenovirus infection. J. Cell. Physiol. 162:26-35.

15. Whyte, P., et al. 1988. Association between an oncogene and an anti-oncogene: the adenovirus E1A proteins bind to the retinoblastoma gene product. Nature. 334:124-129.

16. Bandara, L.R., and La Thangue, N.B. 1991. Adenovirus E1a prevents the retinoblastoma gene product from complexing with a cellular transcription factor. Nature. 351:494-497.

17. Howe, J.A., Mymryk, J.S., Egan, C., Branton, P.E., and Bayley, S.T. 1990. Retinoblastoma growth sup- 
pressor and a 300-kDa protein appear to regulate cellular DNA synthesis. Proc. Natl. Acad. Sci. U. S. A. 87:5883-5887.

18. Guilhot, C., Benchaibi, M., Flechon, J.E., and Samarut, J. 1993. The 12 S adenoviral E1A protein immortalizes avian cells and interacts with the avian RB product. Oncogene. 8:619-624.

19. Faha, B., Harlow, E., and Lees, E. 1993. The adenovirus E1A-associated kinase consists of cyclin E-p33cdk2 and cyclin A-p33cdk2. J. Virol. 67:2456-2465.

20. Shi, Y., et al. 2003. Coordinated histone modifications mediated by a CtBP co-repressor complex. Nature. 422:735-738.

21. Deleu, L., Shellard, S., Alevizopoulos, K., Amati, B., and Land, H. 2001. Recruitment of TRRAP required for oncogenic transformation by E1A. Oncogene. 20:8270-8275.

22. Fuchs, M., et al. 2001. The p400 complex is an essential E1A transformation target. Cell. 106:297-307.

23. Ghosh, M.K., and Harter, M.L. 2003. A viral mechanism for remodeling chromatin structure in G0 cells. Mol. Cell. 12:255-260.

24. Alevizopoulos, K., Catarin, B., Vlach, J., and Amati, B. 1998. A novel function of adenovirus E1A is required to overcome growth arrest by the CDK2 inhibitor p27(Kip1). EMBOJ. 17:5987-5997.

25. Reid, J.L., Bannister, A.J., Zegerman, P., MartinezBalbas, M.A., and Kouzarides, T. 1998. E1A directly binds and regulates the $\mathrm{P} / \mathrm{CAF}$ acetyltransferase. EMBO J. 17:4469-4477.

26. Dorsman, J.C., et al. 1995. The N-terminal region of the adenovirus type $5 \mathrm{E} 1 \mathrm{~A}$ proteins can repress expression of cellular genes via two distinct but overlapping domains. J. Virol. 69:2962-2967.

27. Goodman, R.H., and Smolik, S. 2000. CBP/p300 in cell growth, transformation, and development. Genes Dev. 14:1553-1577.

28. Babiss, L.E., et al. 1986. Mutations in the E1a gene of adenovirus type 5 alter the tumorigenic properties of transformed cloned rat embryo fibroblast cells. Proc. Natl. Acad. Sci. U. S. A. 83:2167-2171.

29. Subramanian, T., Kuppuswamy, M., Nasr, R.J., and Chinnadurai, G. 1988. An N-terminal region of adenovirus E1a essential for cell transformation and induction of an epithelial cell growth factor.
Oncogene. 2:105-112.

30. Chen, D., et al. 2004. Cell-specific effects of RB or $\mathrm{RB} / \mathrm{p} 107$ loss on retinal development implicate an intrinsically death-resistant cell-of-origin in retinoblastoma. Cancer Cell. 5:539-551.

31. Classon, M., and Harlow, E. 2002. The retinoblastoma tumour suppressor in development and cancer. Nat. Rev. Cancer. 2:910-917.

32. MacPherson, D., et al. 2004. Cell type-specific effects of $\mathrm{Rb}$ deletion in the murine retina. Genes Dev. 18:1681-1694.

33. Puri, P.L., et al. 1997. Differential roles of p300 and PCAF acetyltransferases in muscle differentiation. Mol. Cell. 1:35-45.

34. Braun, T., Bober, E., and Arnold, H.H. 1992. Inhibition of muscle differentiation by the adenovirus E1a protein: repression of the transcriptional activating function of the HLH protein Myf-5. Genes Dev. 6:888-902.

35. Tiainen, M., Spitkovsky, D., Jansen-Durr, P., Sacchi, A., and Crescenzi, M. 1996. Expression of E1A in terminally differentiated muscle cells reactivates the cell cycle and suppresses tissue-specific genes by separable mechanisms. Mol. Cell. Biol. 16:5302-5312.

36. Caruso, M., Martelli, F., Giordano, A., and Felsani, A. 1993. Regulation of MyoD gene transcription and protein function by the transforming domains of the adenovirus E1A oncoprotein. Oncogene. 8:267-278.

37. Bagchi, S., Raychaudhuri, P., and Nevins, J.R. 1990. Adenovirus E1A proteins can dissociate heteromeric complexes involving the E2F transcription factor: a novel mechanism for E1A trans-activation. Cell. 62:659-669.

38. Marino, S., Vooijs, M., van Der Gulden, H., Jonkers, J., and Berns, A. 2000. Induction of medulloblastomas in $\mathrm{p} 53$-null mutant mice by somatic inactivation of $\mathrm{Rb}$ in the external granular layer cells of the cerebellum. Genes Dev. 14:994-1004.

39. Vernell, R., Helin, K., and Muller, H. 2003. Identification of target genes of the p16INK4A-pRB-E2F pathway. J. Biol. Chem. 278:46124-46137.

40. Muller, H., et al. 2001. E2Fs regulate the expression of genes involved in differentiation, development, proliferation, and apoptosis. Genes Dev.
15:267-285.

41. Ramaswamy, S., Ross, K.N., Lander, E.S., and Golub, T.R. 2003. A molecular signature of metastasis in primary solid tumors. Nat. Genet. 33:49-54.

42. van ' $t$ Veer, L.J., et al. 2002. Gene expression profiling predicts clinical outcome of breast cancer. Nature. 415:530-536

43. Kraus, M.H., Popescu, N.C., Amsbaugh, S.C., and King, C.R. 1987. Overexpression of the EGF receptor-related proto-oncogene erbB-2 in human mammary tumor cell lines by different molecular mechanisms. EMBO J. 6:605-610.

44. van de Vijver, M.J., et al. 2002. A gene-expression signature as a predictor of survival in breast cancer. N. Engl. J. Med. 347:1999-2009.

45. Spartz, A.K., Herman, R.K., and Shaw, J.E. 2004. SMU-2 and SMU-1, Caenorhabditis elegans homologs of mammalian spliceosome-associated proteins RED and fSAP57, work together to affect splice site choice. Mol. Cell. Biol. 24:6811-6823.

46. Spike, C.A., Shaw, J.E., and Herman, R.K. 2001. Analysis of smu-1, a gene that regulates the alternative splicing of unc-52 pre-mRNA in Caenorhabditis elegans. Mol. Cell. Biol. 21:4985-4995.

47. Isono, K., et al. 2001. Molecular cloning, genetic mapping, and expression of the mouse Sf3b1 (SAP155) gene for the U2 snRNP component of spliceosome. Mamm. Genome. 12:192-198.

48. Charrasse, S., Lorca, T., Doree, M., and Larroque, C. 2000. The Xenopus XMAP215 and its human homologue TOG proteins interact with cyclin B1 to target $\mathrm{p} 34 \mathrm{cdc} 2$ to microtubules during mitosis. Exp. Cell Res. 254:249-256.

49. Gergely, F., Draviam, V.M., and Raff, J.W. 2003. The ch-TOG/XMAP215 protein is essential for spindle pole organization in human somatic cells. Genes Dev. 17:336-341.

50. Soond, S.M., Terry, J.L., Colbert, J.D., and Riches, D.W. 2003. TRUSS, a novel tumor necrosis factor receptor 1 scaffolding protein that mediates activation of the transcription factor NF-kappaB. Mol. Cell. Biol. 23:8334-8344.

51. de Hoog, C.L., Foster, L.J., and Mann, M. 2004. RNA and RNA binding proteins participate in early stages of cell spreading through spreading initiation centers. Cell. 117:649-662. 